\title{
The Abrasive Wear of Gold Jewellery Alloys
}

\author{
H. Heidsiek and M. Clasing
}

Degussa AG., Metals Research Department, Hanau, Federal Republic of Germany

\begin{abstract}
Wear damage to gold jewellery, in particular that caused by abrasive action with clothing or in contact with otber jewellery pieces, is probably the most frequent cause of its premature repair or replacement. One reason for this is undoubtedly the lack of adequate understanding throughout the industry regarding the mechanisms of wear, and the factors which can cause an improvement in the wear resistance of gold jewellery alloys.
\end{abstract}

Since processes involving friction and wear are commonly encountered problems leading to sudden or gradual failure of industrial components and assemblies they have been the subject of many investigations during recent years. It has emerged from these that all wear phenomena are due to a number of rather complex processes in or at the surface of solids and satisfactory physical understanding of these has still to be reached (1-6). This also appears to be the case, for example, in respect of the wear behaviour, which has been much studied, of mating gold surfaces in electrotechnical or electronic applications such as sliprings and brushes, connectors, switches or other contact systems.

The literature regarding the wear of jewellery products is sparse, by contrast, and embodies a number of poorly supported hypotheses. Thus it is still widely assumed that the wear resistances of gold jewellery alloys are primarily a function of their hardnesses. It is the objective of this article to contribute to a better metallurgical understanding of the wear of gold jewellery alloys particularly in respect of this correlation between hardness and wear resistance. The data reported have resulted from an investigation of various common gold jewellery alloys, in which the abrasive wear resistance has been quantitatively measured as a function of different microstructural states of specimens expressed in terms of different values of hardness.

\section{Definitions of Wear}

Tribology, the science of friction and wear phenomena, is a comparatively recent area of metallurgy and thus far no complete commonly accepted definition of wear has been agreed upon. Nevertheless some suggestions in this respect have been widely accepted (4). Two suggested definitions are:

(1) The removal of material from surfaces in relative motion by mechanical and/or chemical processes (Tabor).

(2) The progressive loss of substance from the operating surface of a body occurring as a result of relative motion of the surface (OECD).

In expanding the OECD definition the German Standard DIN 50320 defines wear to be:
The progressive loss of substance from the surface of a solid body due to mechanical action, that is contact and relative motion of a solid, liquid or gaseous counterbody.

\section{A supplement defines tribological action as:}

The action on the surface of a solid body by contact and relative motion of a solid, liquid or gaseous counterbody.

These definitions will already have given the reader the impression that wear is a complex phenomenon. This impression can be easily confirmed by considering two different tribological systems involved in typical jewellery products.

Let us take a chain of high carat gold alloy and a wrist-watch bracelet of the same gold alloy. In the first case two metallic bodies, that is two chain-links, are involved in tribological actions, while in the case of the bracelet, the counterbody will be principally sleeve cloth or hard particles, for example dust, in the interface. Although the same alloy is subject to wear in each situation the appearance of the alloy wear is different in the two cases since the tribological systems are different.

This example illustrates that it is not so much the properties of a single partner of a wear system, for example those of the gold alloy, that determine the process of wear, as the properties of the whole tribo-system which involves:

(a) The elements, for example two or more mating bodies, the interfacial medium, the surrounding medium, etc.

(b) The properties of the elements, for example the type of materials constituting the elements, their geometries etc.

(c) The interactions between the elements determined by the contact and friction conditions, and the wear mechanisms.

These considerations indicate that in contrast to characteristic material-related properties such as electrical conductivity, tensile strength, hardness, etc. wear occurring under tribological action is always a result of the interaction of different structural parts or technical components which can only be described by system-related wear characteristics. This hypothesis leads directly to the conclusion that

Wear resistance is not an intrinsic mechanical property; each wear event must be considered as a feature of a tribological system (3). 


\section{Mechanisms of Wear}

German Standard DIN 50320 identifies four mechanisms as controlling the process of wear in any tribological system, namely, adhesion, abrasion, surface fatigue and tribochemical reactions.

Since abrasion is often the most destructive of these mechanisms causing the greatest material loss in the shortest times, we shall restrict discussion in this article to abrasive wear.

\section{Definition of Abrasive Wear}

In abrasive wear hard particles enter the surface of a softer body and remove material from the surface by ploughing or micro-cutting ('scratching'). Abrasion can be quantitatively measured by a specific wear rate $\dot{w}$ which is defined as:

$$
\dot{w}=\frac{\Delta m}{s A \rho} \text { often expressed as } \frac{\mu \mathrm{m}}{\mathrm{m}} \times 10^{6}
$$

where

$$
\begin{aligned}
\Delta m & =\text { material loss due to wear } \\
s & =\text { sliding distance } \\
A & =\text { apparent contact area } \\
\rho & =\text { specific density of the worn material. }
\end{aligned}
$$

Although $\dot{w}$ is a dimensionless quantity it is often more convenient to use $\dot{w}$ in the form of $\mu \mathrm{m} / \mathrm{m} \times 10^{6}$, since then $\dot{w}$ indicates the thickness in micrometres of the surface layer which will be removed per metre of sliding distance.

Based on the above definition of wear rate $\dot{w}$, we are now able to define specific abrasive wear resistance $\dot{w}^{-1}$, as the reciprocal value of the wear rate $\dot{w}$. In the form $\mathrm{m} / \mu \mathrm{m}$ it indicates the distance (in metres) over which sliding must occur before a surface layer of $1 \mu \mathrm{m}$ thickness is worn away from the surface of an element in the system. In the figures discussed below the wear resistance $\dot{w}^{-1}$ will always be considered as a function of the sliding distance.

Two basic mechanisms can be distinguished during the removal of metal in abrasive wear: during micro-cutting, material is directly removed from a worn surface by hard particles. In this case the volume of removed material is equal to the volume of the wear grooves (scratches). Frequently, however, only part of the material equivalent to the volume of the wear grooves is actually removed since, as a result of plastic deformation, bulges of material are formed at the edges of the grooves. This tribological action is called micro-ploughing. These phenomena are readily illustrated in Figure 1 which shows a typical scanning electron micrograph of the surface topography of a link in a flexible 14 carat rolled-gold wristwatch strap which had been in use for several years. The topography indicates that this surface has been subject to abrasive wear: micro-cutting has occurred at area ' $A$ ', and micro-ploughing can be identified easily by the bulges at the edges of the wear groove at area ' $B$ '.

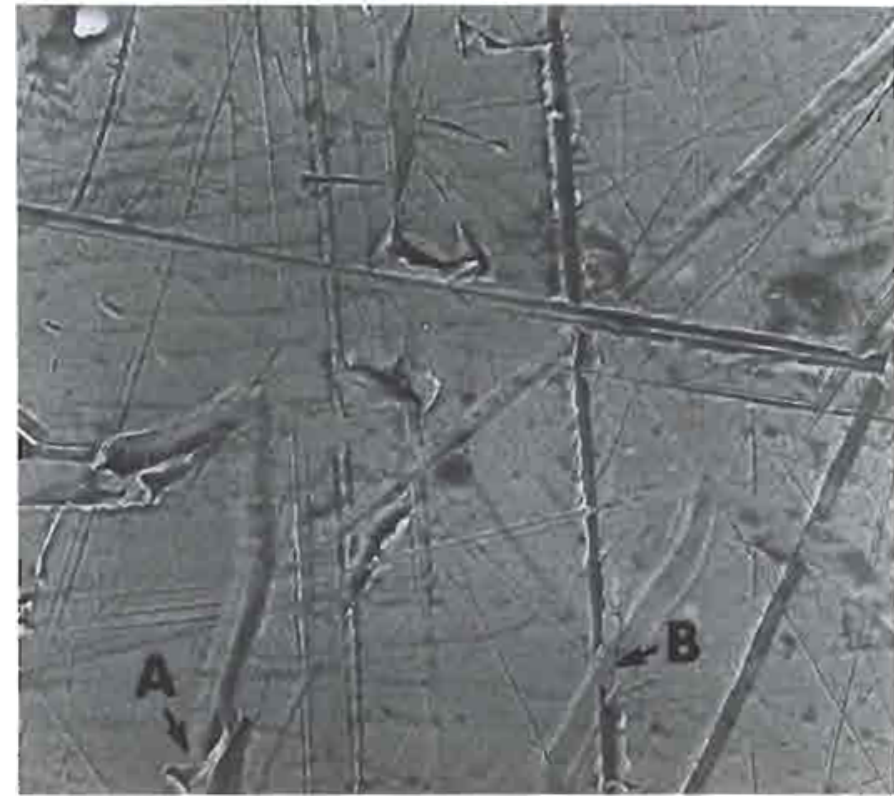

Fig. 1 Scanning electron micrograph of the topography of a link of a $14 \mathrm{ct}$. rolled gold wrist watch strap after several years' use

$\times 1000$

\section{Experimental Studies}

\section{Equipment and Procedures}

In the experiments reported below samples of a standard gold jewellery alloy, gold $/ 26$ silver $/ 15.5$ copper per cent, in various metallurgical conditions yielding different hardnesses, have been subjected to abrasive wear using abrasive cloth under controllable conditions of load, traverse distance and velocity, and test atmosphere. The effects of hardening additives and the behaviour of other gold alloys have also been studied.

The tribometer used is shown in Figure 2(a): specimens of $10 \times 10 \times 2 \mathrm{~mm}$ in size were fixed in the specimen holder and reciprocated linearly over an abrasive counterpart, for example abrasive paper, microcut-polishing cotton or cloth at a variable velocity $\nu$ (average 0.1 to $0.8 \mathrm{~m} / \mathrm{s}$.). The length of a single sliding track could be varied between 10 and $40 \mathrm{~cm}$. For the present investigations a track of $40 \mathrm{~cm}$ was chosen and this length held constant. Different specimen loads $F_{N}=1.25$ to $20 \mathrm{~N}$ could be applied.

Since the heights of the asperities of the abrasive material decrease with increasing sliding distance leading to a decreasing severity of the abrasive counterpart, the paper or cloth was pulled forward after a given sliding distance (feed $f$ ) in order to supply virgin abrasive, as indicated schematically in Figure 2(b). Thus reproducible wear conditions were ensured even over long sliding distances. The measurements could be carried out in dry as well as in liquid atmospheric conditions (for example artificial perspiration). 

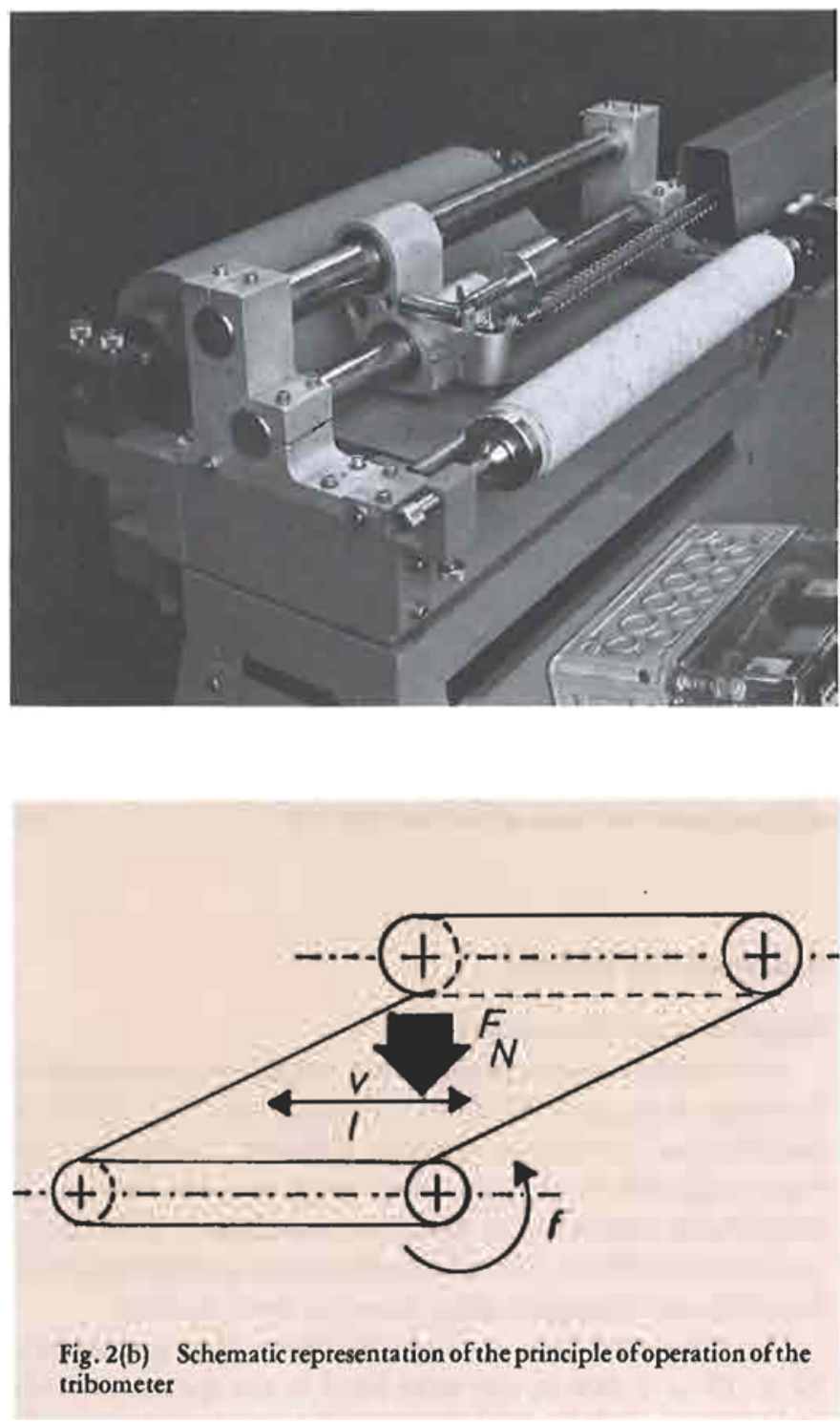

Since commercially available abrasive papers commonly used for similar investigations of abrasive wear proved to be too harsh for the present tests the measurements reported below were carried out with 'microcut', a polishing cotton normally used in metallographical microsection preparation. Figure 2(c) shows a scanning electron micrograph of the 'microcut' topography and reveals small abrasive, silicon carbide particles $(\leq 15 \mu \mathrm{m})$ having a rather low distribution density. Figure 2 (d) shows the surface of a 14 carat gold alloy after being worn on the tribometer. Comparison with the worn article shown in Figure 1 reveals that the appearance of wear is the same in the two cases and that the severity of practical wear is quite well simulated by the selected test abrasive medium.
Fig 2(a) Photograph of the tribometer used in this work, with the continuous abrasive cloth and loaded specimen clearly visible

\section{Standardization Tests}

The influence of load $F_{N}$ and abrasive feed $f$ upon wear rate were first investigated in detail. The measurements were carried out by testing a 14 carat gold alloy (gold $/ 26$ silver $/ 15.5$ copper per cent) which had been age hardened for one hour at $350^{\circ} \mathrm{C}$ to a Vickers hardness of $180 \mathrm{HV}$.

In Figure 3(a) the wear resistance $\dot{w}^{-1}$ of this alloy is plotted as a function of the sliding distance. For the relevant experiments the load $F_{N}$ was varied between 5 and $20 \mathrm{~N}$ while other parameters such as sliding velocity $(v=0.5 \mathrm{~m} / \mathrm{s})$, sliding track length $(l=0.4 \mathrm{~m})$ and feed $(f=4 \mathrm{~m})$ were kept constant. The following features of the results are shown in Figure 3(a):

(i) For $F_{N}=20 \mathrm{~N}$ the wearing-in behaviour indicated by an initial increase in $\dot{w}^{-1}$ was terminated after a few metres, while for $F_{N}=5 \mathrm{~N}$ the wear resistance continuously increased even after $250 \mathrm{~m}$

(ii) With increasing load the final wear resistance decreased.

The second experimental parameter, the feed $f$, determines the length of the sliding track used before the abrasive material was moved forward in order to supply a virgin abrasive area.

The effect of varying feed is shown in Figure 3(b) in which $\dot{w}^{-1}$ is plotted again against sliding distance. The most stringent conditionsoccur with $f=0.8 \mathrm{~m}$ when the specimen was always worn against virgin abrasive leading to a very small wear resistance $\left(\dot{w}^{-1}=1.2 \mathrm{~m} / \mu \mathrm{m}\right)$ and almost negligible wearingin behaviour. With increasing feed, however, that is with the specimen being worn by the same abrasive area for longer distances (for example 4 or $20 \mathrm{~m}$ ) an apparent increase of the wear resistance is observed. Thus for $f=20 \mathrm{~m}$, for instance, the wearing-in behaviour was not terminated, even after $250 \mathrm{~m}$ sliding and it can be assumed that the final wear resistance which would be achieved would be about four times greater than that obtained with $f=0.8 \mathrm{~m}$.

In order to observe the wearing-in behaviour with sufficient accuracy, and to reach a stable wear resistance within reasonable sliding distances, as well as to guarantee reproducible results, a load $F_{N}$ of $10 \mathrm{~N}$ and a feed $f$ of $4 \mathrm{~m}$ were selected for the investigation of the correlation of gold alloy hardness and abrasive wear resistance.

\section{Metallurgical Treatment and Wear Resistance}

According to Rapson and Groenewald (7) the hardness of the 14 carat gold alloy (gold $/ 26$ silver $/ 15.5$ copper) can be increased by isothermal annealing at $300^{\circ} \mathrm{C}$. It is known that this increase in hardness, which is caused by ordering $(\mathrm{AuCu})$ and precipitation $(\mathrm{AgCu})$, can be further increased by preceding cold working, as well as by addition of grain refining elements. 


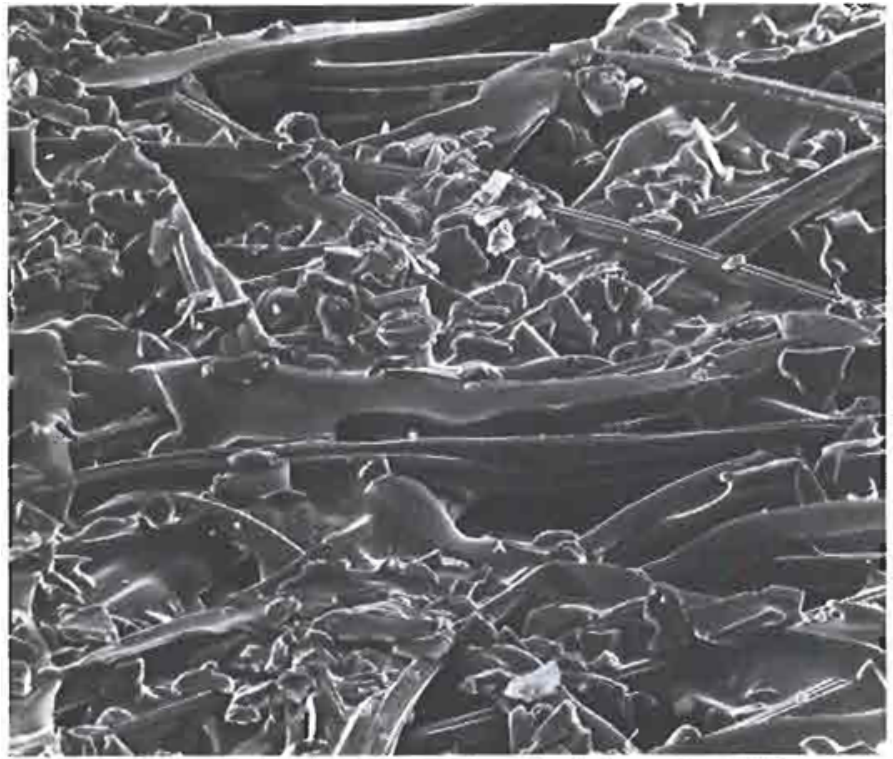

Fig. 2(c) Scanning electron micrograph of the 'microcut' polishing cotton used in this experiment showing small silicon carbide particles

Thus different thermal and mechanical treatments have been applied to the specimens prior to the measurement of the wear resistance. These measurements were carried out with the above parameters, load and feed, held constant at the levels determined in the standardization test. The following results, which are summarized in Table I, were obtained:

(1) The soft state was achieved by annealing the alloy for one hour at $750^{\circ} \mathrm{C}$ and subsequently water quenching. In this state the alloy had a Vickers hardness of $120 \mathrm{HV}$ and showed a wear resistance of $2.43 \mathrm{~m} / \mu \mathrm{m}$.

(2) The age-hardened state was established by quenching from a temperature of $750^{\circ} \mathrm{C}$ and subsequently isothermal annealing for one hour at $350^{\circ} \mathrm{C}$. This treatment led to a Vickers hardness of $237 \mathrm{HV}$ and resulted in a wear resistance of $2.07 \mathrm{~m} / \mu \mathrm{m}$.

(3) The cold-worked state which was established by 70 percent cold-rolling was characterized by a Vickers hardness of $272 \mathrm{HV}$ and a measured wear resistance of $1.94 \mathrm{~m} / \mu \mathrm{m}$.

(4) The annealed state after cold - deformation was achieved by annealing the cold-worked specimen above for three hours at $280^{\circ} \mathrm{C}$. This procedure lead to a Vickers hardness of $312 \mathrm{HV}$ and a wear resistance of $2.17 \mathrm{~m} / \mu \mathrm{m}$.

The influence of typical 'hardening' additives such as cobalt, yttrium and zirconium upon hardness and wear resistance of the gold alloy under investigation has also been examined. These results are also summarized in Table $\mathrm{I}$ :

(5) The addition of 0.7 weight per cent of cobalt increased the hardness of the alloy significantly from $120 \mathrm{HV}$ to $168 \mathrm{HV}$ in the soft state and from $237 \mathrm{HV}$ to even $341 \mathrm{HV}$ in the

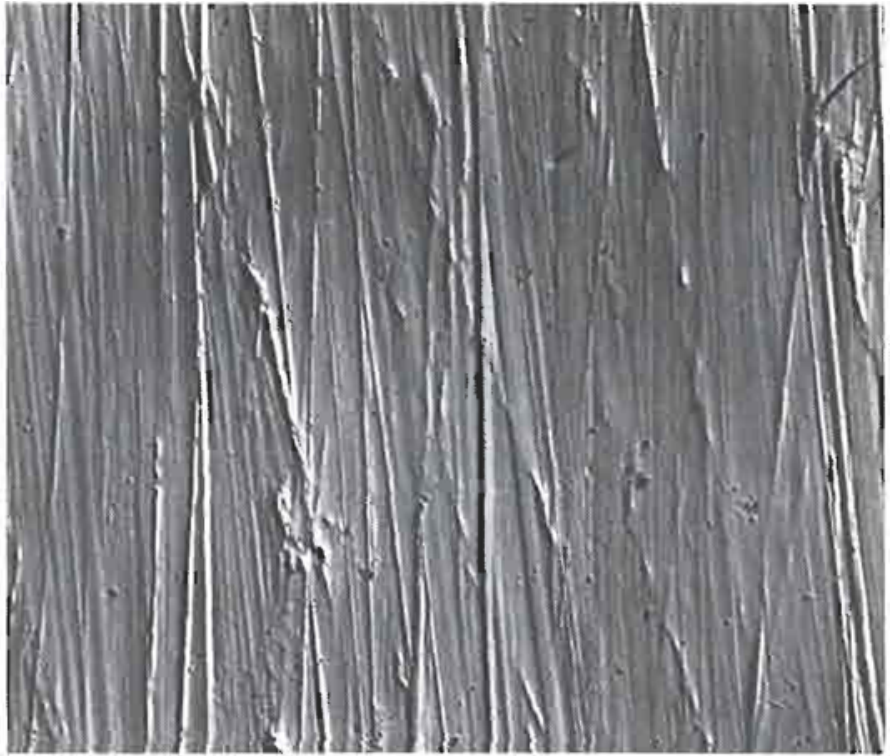

Fig 2(d) Scanning electron micrograph of a $14 \mathrm{ct}$. gold alloy after wear experiments on the tribometer

annealed state. Surprisingly, the wear resistance did not follow this trend: with $\dot{w}^{-1}=2.19$ and 2.05 , respectively, this alloy is actually slightly less wear resistant than the cobalt-free alloy.

(6) The addition of 0.25 weight per cent of yttrium and 0.39 weight per cent of zirconium improved the wear resistance. A comparison with the standard alloy showed an increase of 30 per cent for the cold worked state, although the increase of hardness was less significant than with cobalt.

\section{Discussion}

Before the foregoing results are discussed in detail some general remarks concerning the experimental conditions should be made. The standardization measurements have demonstrated that the wear resistance of an alloy depends upon system parameters such as the load applied during the tribological action (see Figure 3(a)) as well as upon the length of the sliding track after which virgin abrasive areas are supplied. Figure 3(b) shows an apparent increase of the wear resistance occurring with increasing feed $f$. This must be attributed, however, to the decreasing asperity height of the abrasive material.

Clearly, the type and severity of the abrasive have a positive effect upon the absolute value of the wear resistance of an alloy and the choice of abrasive material is very important. Preliminary investigations with commercially available abrasive paper (1 200 grit), as well as with plain cotton, have shown that the asperity of even the finestabrasive paper causes wear which is far too severe for meaningful comparisons in these tests. The 


\section{Table I}

Abrasive Wear Resistance, $\dot{\mathrm{w}}^{-1}$, of a 14 Carat Gold Alloy in Different Metallurgical Siates Measured after $250 \mathrm{~m}$ Sliding Distance

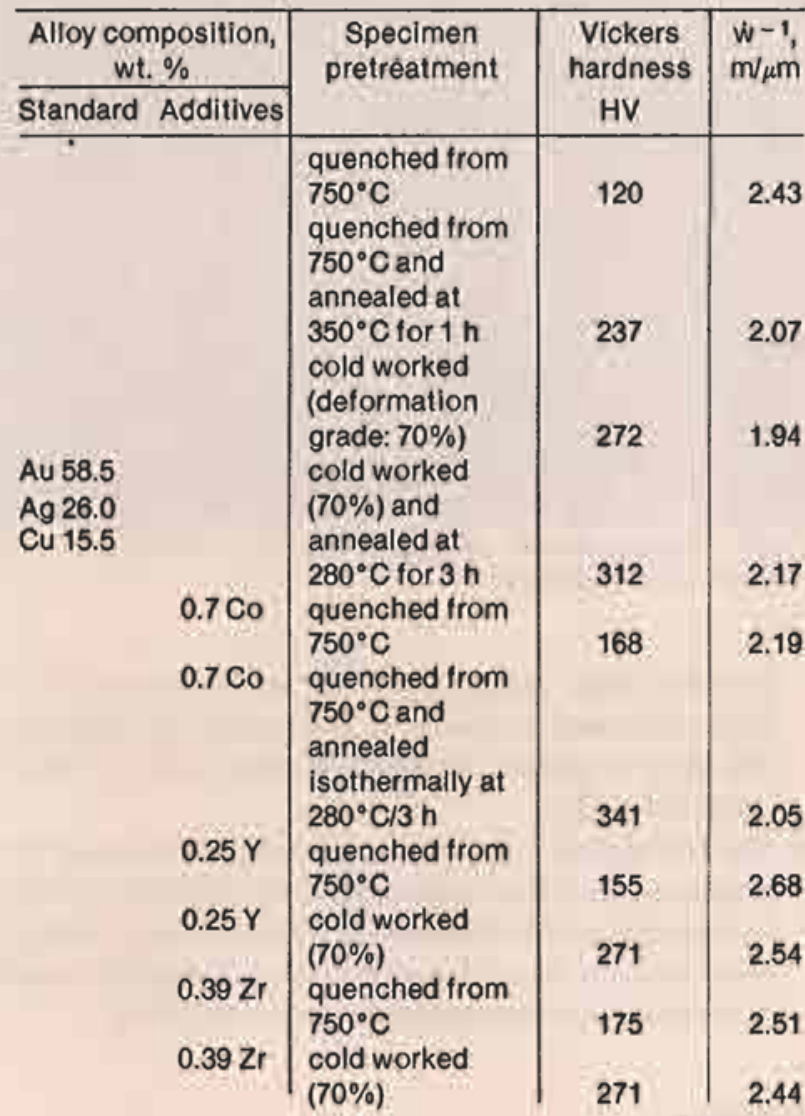

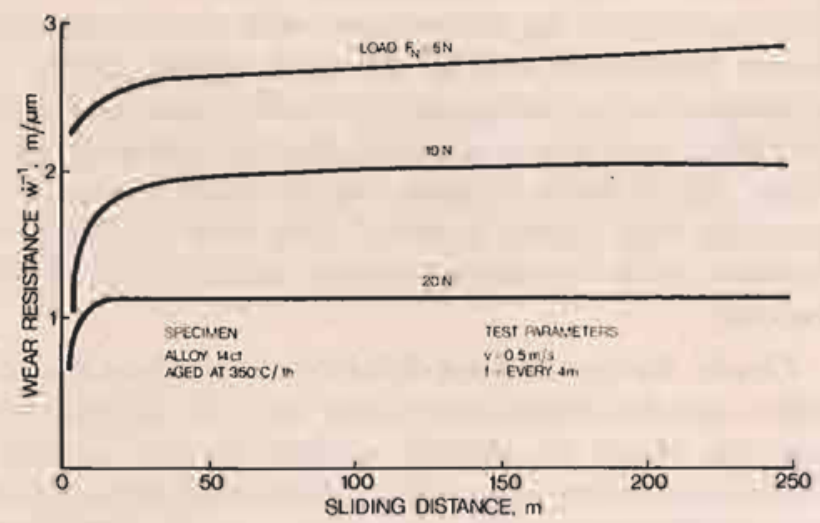

Fig. 3(a) Influence of the applied load upon the wear resistance of a $14 \mathrm{ct}$. reference alloy

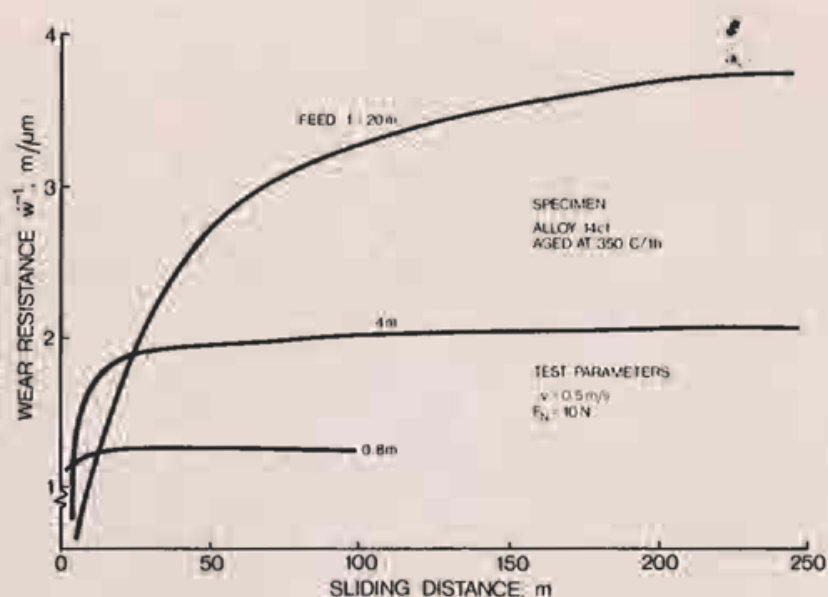

Fig. 3(b) Influence of the feed of virgin abrasive upon the wear resistance of a $14 \mathrm{ct}$. reference alloy

typical running-in behaviour as illustrated in Figures 3(a) and (b) was not detectable. On the other hand the abrasive attack of plain cotton was far too small and a measurable loss in mass was detectable only over very long sliding distances. Additionally, the wear mechanism of this tribo-system appeared to be surface fatigue rather than abrasion. In order to improve the abrasive characteristics the cotton was additionally dusted with small amounts of standardized test-dust. Since the dust could not be constantly supplied, however, the resulting measurements were not very reproducible and varied widely.

Thus, the most reliable measurements were achieved with 'microcut' polishing cotton although the hardness of approximately $3000 \mathrm{HV}$ of the silicon carbide particles contained therein might be expected to be too high to be able to simulate a 'real' abrasive wear attack. The relative hardness of each abrasive seems to be of little importance, however, since the hardness of even softer 'natural' dust particles like corundum, feldspar or quartz is generally at least five times higher than the hardness of the investigated alloys. Thus it does not seem likely that the wear mechanism has been fundamentally changed by using silicon carbide-based abrasive material. It seems plausible to assume that the moderate wear attack of 'microcut' is due to the low distribution density (of abrasive particles per $\mathrm{cm}^{2}$ ) of the material leading to a surprisingly good teproducibility of the abrasive wear measurements.

Summarizing the standardization test results, it can be concluded that these measurements have confirmed the system character of wear. Consequently, the data presented in this paper cannot be taken as absolute values but must be seen as relative results. 


\section{Hardness-Wear Resistance Correlation}

According to Table 1 the hardness of the gold-silver-copper alloy can be varied between $120 \mathrm{HV}$ (softstate) and $312 \mathrm{HV}$ (cold worked and annealed state). This increase of 250 per cent, however, is not reflected in the corresponding wear resistance data. On the contrary: the wear resistance of the hard state (2.17 $\mathrm{m} / \mu \mathrm{m})$ is about 10 per cent smaller than the wear resistance of the soft state $(2.43 \mathrm{~m} / \mu \mathrm{m})$. This result does not agree with the commonly accepted correlation but it is confirmed by the other measurements teported in Table 1 which show that when using additions of cobalt, yttrium and zirconium the soft state is more abrasive wear resistant then the hard state. These results demonstrate that hardness is not a suitable indicator for the abrasion wear resistance of this alloy, since although the alloy with 0.7 weight per cent cobalt has a Vickers hardness of $341 \mathrm{HV}$ in the fully annealed state the corresponding wear resistance appears to be only $2.05 \mathrm{~m} / \mu \mathrm{m}$ while for the soft cobalt-free alloy a wear resistance of $2.43 \mathrm{~m} / \mu \mathrm{m}$ at a hardness of $120 \mathrm{HV}$ is attained. Furthermore, the highest observed wear resistance of $2.68 \mathrm{~m} / \mu \mathrm{m}$ occurs with the soft state $(155 \mathrm{HV})$ of the alloy with 0.25 weight per cent yttrium.

These results are further confirmed by two other alloys of different composition which have been tested separately. The results are summarized in Table 11 . The 18 carat alloy gold $/ 25$ copper per cent was chosen in order to study the influence of the ordered $\mathrm{AuCu}$ phase upon the wear resistance of the alloy. It emerged that the ordered state with a hardness of $356 \mathrm{HV}$ was again less wear resistant than the corresponding soft state. This was also true for a rose-coloured 14 carat gold/ 36 copper $/ 5.5$ nickel per cent alloy which had a wear resistance of $3.5 \mathrm{~m} / \mu \mathrm{m}$ for the soft state although its hardness differs only slightly from the corresponding hardness of the investigated alloy of Table I.

These resultsclearly show that a hard alloy cannot be assumed to be automatically wear resistant. This can be easily understood, however, by knowing the two basic wear mechanisms of abrasion: micro-cutting and micro-ploughing. The wear rate, that is the amount of material which is removed from a worn surface per unit sliding distance, is mainly determined by the prevailing wear mechanism. The amount of removed material, and consequently the wear rate, is high if micro-cutting prevails since this mechanism leads to an immediate material loss. During micro-ploughing, however, the surface is primarily plastically deformed which does not cause an immediate material loss. Although there is no doubt that the hardness of a material determines whether or not hard abrasive particles can penetrate a surface, other material properties like ductility, toughness or brittleness also strongly influence the wear resistance of an alloy.

The higher the ability of a surface to absorb plastic deformation, the higher will be the portion of micro-ploughing, the lower will be the wear rate and, conversely, the higher will be the
Table II

Abrasive Wear Resistance, $\dot{w}^{-1}$ of 18 and 14 Carat Gold Alloys in Various Metallurgical States

\begin{tabular}{|c|c|c|c|}
\hline $\begin{array}{c}\text { Alloy composition, } \\
\text { wt. } \%\end{array}$ & $\begin{array}{l}\text { Specimen } \\
\text { pretreatment }\end{array}$ & $\begin{array}{c}\text { Vickers } \\
\text { hardness HV }\end{array}$ & $\begin{array}{l}\dot{w}-1, \\
m / \mu m\end{array}$ \\
\hline $\begin{array}{l}\text { Au } 75 \\
\text { Cu } 25\end{array}$ & $\begin{array}{l}\text { quenched from } \\
750^{\circ} \mathrm{C} \\
\text { cold worked } \\
(70 \%) \text { and } \\
\text { annealed at } \\
300^{\circ} \mathrm{C} \text { for } 1 \mathrm{~h}\end{array}$ & 155 & $\begin{array}{l}2.25 \\
2.15\end{array}$ \\
\hline $\begin{array}{l}\text { Au } 58.5 \\
\text { Cu } 36.0 \\
\text { Ni } 5.5\end{array}$ & $\begin{array}{l}\text { quenched from } \\
750^{\circ} \mathrm{C} \\
\text { cold-worked } \\
(70 \%)\end{array}$ & $\begin{array}{l}126 \\
280\end{array}$ & $\begin{array}{l}3.49 \\
3.33\end{array}$ \\
\hline
\end{tabular}

wear resistance of the alloy.

These considerations may explain why the soft states of the investigated alloys turned out to be more wear resistant than the hard states, and why no correlation between hardness and wear resistance has been found.

\section{Concluding Remarks}

Fundamental aspects of abrasive wear have been reviewed. Quantitative' wear measurements have been carried out with a 14 carat gold $/ 26$ silver $/ 15.5$ copper per cent alloy using a specially designed tribometer. The alloy was treated to yield different microstructural states giving different hardnesses. It was found that for this gold alloy in the tribological conditions used an increased hardness of the alloy did not indicate a corresponding increase of its abrasive wear resistance. The soft state of the investigated alloy appeared to be slightly more wear resistant than harder states.

\section{Acknowledgements}

The authors wish to express their thanks to Dipl.-Ing. (FH) G. Jäckel and Dipl.-Ing. (FH) H. Schmidt for preparing the specimens and carefully measuring the weat resistance and hardness, as well as to the International Gold Corporation for financial support.

\section{References}

1 E. Hornbogen, 'Metallurgical Aspects of Wear' Fortschrifteberichteder VDI Zeitschriften, 1976, Serie 5, No. 24

2 K.H. ZumGahr, Z. Metallkd., 1982, 73, 267

3 K.H. ZumGahr, Met. Prog., 1979, 46

4 T.S. Eyre, Powder Metall., $1981,2,57$

5 E. Rabinowicz, 'Friction and Wear of Materials', John Wiley, New York, 1965

6 E. Rabinowicz, Mater. Sci. Eng., 1976, 25, 23

7 W.S. Rapson and T. Groenewald, 'Gold Usage', Academic Press, 1978, p. 38 